\title{
Lung ultrasound findings in pediatric patients with COVID-19
}

\author{
Carmina Guitart ${ }^{1,2} \cdot$ Ricardo Suárez $^{1,2} \cdot$ Mònica Girona $^{1,2} \cdot$ Sara Bobillo-Perez ${ }^{1,2}$ - Lluïsa Hernández ${ }^{1,2}$. \\ Mònica Balaguer ${ }^{1,2}$ - Francisco José Cambra ${ }^{1,2} \cdot$ Iolanda Jordan $^{2,3} \cdot$ On behalf of the KIDS-Corona study group, Kids \\ Corona Platform
}

Received: 6 August 2020 / Revised: 12 September 2020 / Accepted: 9 October 2020 / Published online: 22 October 2020

(C) Springer-Verlag GmbH Germany, part of Springer Nature 2020

\begin{abstract}
During the pandemic caused by the novel coronavirus (COVID-19), lung ultrasound has been used to diagnose and monitor respiratory condition. The aim of the study was to describe lung ultrasound findings in children with a COVID-19 infection. Patients younger than 18 years old and positive for COVID-19, admitted to pediatric tertiary referral hospital were included. They were divided into two groups depending on the presence of respiratory symptoms. Lung ultrasound results were categorized into four degrees according to Soldati et al. score (J Ultrasound Med 39:1-7,2020) and it was also described the presence and type of consolidation. Sixteen patients were recruited. The median age was 11 years old (IQR 2.8-12). Four children (25\%) required admission to the intensive care unit. Six patients (37.5\%) presented with respiratory symptoms. Most of them showed S.score of 2 and subpleural consolidations were observed in four cases $(66.6 \%)$. Ten patients $(62.5 \%)$ presented with non-respiratory symptoms, lung ultrasound showed S.score from 0 to 2 . Three (30\%) were diagnosed of multisystem inflammatory syndrome and lung ultrasounds showed S.score of 2 with bilateral pleural effusion.

Conclusions: Children with COVID-19 and respiratory symptoms mostly showed a S.score of 2 and 3 with subpleural consolidations, upon the lung ultrasound assessment.

What is Known:

- Lung ultrasound is a useful tool for monitoring patients with respiratory symptoms in both adults and children. Lung ultrasounds are altered in adult patients with COVID-19.

What is New:

- Lung ultrasound might improve COVID-19 assessment, it could be a useful tool to diagnose and monitor patients throughout the COVID-19 pandemic. Even COVID-19 patients with non-respiratory symptoms have lung alterations that are visible on lung ultrasound.
\end{abstract}

Communicated by Piet Leroy

Electronic supplementary material The online version of this article (https://doi.org/10.1007/s00431-020-03839-6) contains supplementary material, which is available to authorized users.

Mònica Balaguer

mbalaguer@sjdhospitalbarcelona.org

Carmina Guitart

cguitart@sjdhospitalbarcelona.org

Ricardo Suárez

rsuarez@sjdhospitalbarcelona.org

Mònica Girona

mgirona@sjdhospitalbarcelona.org

Sara Bobillo-Perez

sbobillo@fsjd.org

Lluïsa Hernández

lhernandez@sjdhospitalbarcelona.org
Francisco José Cambra

fjcambra@sjdhospitalbarcelona.org

Iolanda Jordan

ijordan@sjdhospitalbarcelona.org

1 Pediatric Intensive Care Unit, Hospital Sant Joan de Déu, Universitat de Barcelona, Passeig de Sant Joan de Déu, 2, Esplugues de Llobregat, 08950 Barcelona, Spain

2 Disorders of Immunity and Respiration of the Pediatric Critical Patient Research Group, Institut Recerca Hospital Sant Joan de Déu, Universitat de Barcelona, Barcelona, Spain

3 Pediatric Infectious Diseases Research Group, Institut Recerca Hospital Sant Joan de Déu, CIBERESP, Barcelona, Spain 
Keywords COVID-19 · Lung ultrasound · Pneumonia · Pediatrics

$\begin{array}{ll}\text { Abbreviations } \\ \text { CT } & \text { Computed tomography } \\ \text { CXR } & \text { Chest X-ray } \\ \text { ELISA } & \text { Enzyme-linked immunosorbent assay } \\ \text { LUS } & \text { Lung ultrasound } \\ \text { MIS-C } & \text { Multisystem inflammatory syndrome in children } \\ \text { PCR } & \text { Real-time polymerase chain reaction } \\ \text { PICU } & \text { Pediatric intensive care unit } \\ \text { PRISM III } & \text { Pediatric Risk of Mortality Score III } \\ \text { UTI } & \text { Urinary tract infection }\end{array}$

\section{Introduction}

In recent years, lung ultrasound (LUS) has emerged as a bedside tool that is useful to identify and monitor many respiratory pathologies $[2,3]$, especially in intensive care units.

When the pandemic related to the novel coronavirus (COVID-19) emerged in China in December 2019, computed tomography (CT) was initially recommended in order to detect early alterations in infected patients and to monitor lung changes [4]. A recent article suggested that children present milder forms of COVID-19 than adults, and therefore, the use of CT scans should be limited in this population [5]. Chest Xray $(\mathrm{CXR})$ is also a routinely used tool for diagnosing respiratory diseases, including COVID-19 pneumonia, but it has relatively low sensitivity and accuracy [6]. For these reasons, LUS has been increasingly used for the diagnosis of respiratory conditions, including COVID-19 [6, 7]. In a recent Chinese report reflecting a study performed on adults, LUS features were related to the severity of the disease [8]. Moreover, CT and LUS have been compared and have shown a good correlation [9]. Both CT and LUS have high sensitivity to diagnose COVID-19 pneumonia [10]. In those cases with hemodynamic and respiratory instability or with high contagiousness of COVID-19, LUS could be the best option due to its easy bedside accessibility. Moreover, LUS does not use ionizing radiation and is less expensive than the CT scan. Some ultrasound patterns are currently being identified in COVID-19 patients, and having the ability to define and recognize these patterns might be helpful in determining which patients are most at risk for respiratory failure, depending on the severity of LUS alterations [11]. What's more, when LUS is performed by trained clinicians, it may help to stratify the risk of respiratory failure in suspected COVID-19 patients [12]. Despite most of the available information referring to the adult population [7], there are recent studies that describe the use of LUS in children with COVID-19 [13].

The aim of the study was to describe LUS patterns in children with a COVID-19 infection and respiratory symptoms and LUS patterns in COVID-19 patients with nonrespiratory symptoms. A secondary objective was to analyze if the LUS pattern correlated with the severity.

\section{Materials and methods}

This was a prospective observational study performed at the Hospital Sant Joan de Déu, a pediatric tertiary referral hospital. Patients younger than 18 years old admitted to the hospital with a positive real-time polymerase chain reaction (PCR) result for COVID-19 or a positive COVID-19 IgM/IgG enzyme-linked immunosorbent assay (ELISA) result were included. PCR was carried out in accordance with the European Centre for Disease Prevention and Control standards, using nasopharyngeal swabs. The study was approved by the institutional review board and by the local Ethics Committee, and parental informed consent was required. The research study followed the Declaration of Helsinki recommendations.

For each patient, we obtained data on the age at admission, sex, severity as measured by the Pediatric Risk of Mortality Score III [PRISM III] [14], length of pediatric intensive care unit (PICU) and hospital stay, respiratory and hemodynamic support needed, and mortality.

Respiratory symptoms were described as the presence of tachypnea, shortness of breath, and/or cough. Acute respiratory failure was defined as a $\mathrm{PaO} 2$ of $<60 \mathrm{mmHg}$ or an $\mathrm{SaO} 2$ of $<88 \%$ on room air and/or an acute increase in $\mathrm{PaCO} 2$ above $50 \mathrm{mmHg}$ [15]. Multisystem inflammatory syndrome in children (MIS-C) was defined following the WHO case definition [16].

\section{LUS performance}

Intensivists, who have received standard training in LUS (Winfocus PNCUS BL1P) and with at least 3 years of experience with LUS technique, performed the examination. The intensivist wore the appropriate personal protective equipment. The LUS was carried out at the patient's bedside, using a 12-MHz lineal probe, screening the six pulmonary sections following the Via G [17] procedure (superior an inferior sections in parasternal, anterolateral, and posterior areas) [17-19]. In order to minimize the spread of the virus and other microbes, LUS was performed with the probe protected by a single-use plastic cover, and an ultrasound transmission gel in a single-use package. After the examination, all the material was cleaned correctly $[1,20]$.

Even though there is currently no consensus available on classifying the degree of aeration loss and pattern [21], lung 
aeration and lung pattern were classified following the score proposed by Soldati et al. [1] for COVID-19 patients (score 0: normal lung sliding, regular pleural line and A-lines; score 1: vertical artifacts are described, pleural line indented with several B-lines; score 2: broken pleural line with dark and with consolidation areas; score 3 large regions of white lung) [1]. It was also describe the presence and type of consolidation (subpleural consolidations $<1 \mathrm{~cm}$, consolidation with the "shred sign" and bronchogram, and consolidation with a tissue-like pattern without bronchogram) [22-25]. Figure 1 shows how the patients were classified based on the LUS findings as regards aeration loss and lung patterns. More images are shown in the supplementary material.

A statistical analysis was performed using the IBM SPSS 25.0 Statistics ${ }^{\circledR}$ program. Categorical variables were indicated as frequency $(n)$ and percentage (\%), whereas continuous variables were summarized as median and interquartile range (IQR). The comparison of categorical variables was performed using the $\chi 2$ test or Fisher's exact test. Continuous variables were compared with the Mann-Whitney $U$ test. Probability values of less than 0.05 were considered statistically significant.

\section{Results}

Sixteen patients were recruited during the study period. The patients' median age was 11 years old (IQR 2.8-12) and 11 (68.8\%) cases were males. Fifteen patients had positive PCR for COVID-19. Only one patient with MIS-C had positive
Fig. 1 Classification of patients based on the LUS findings

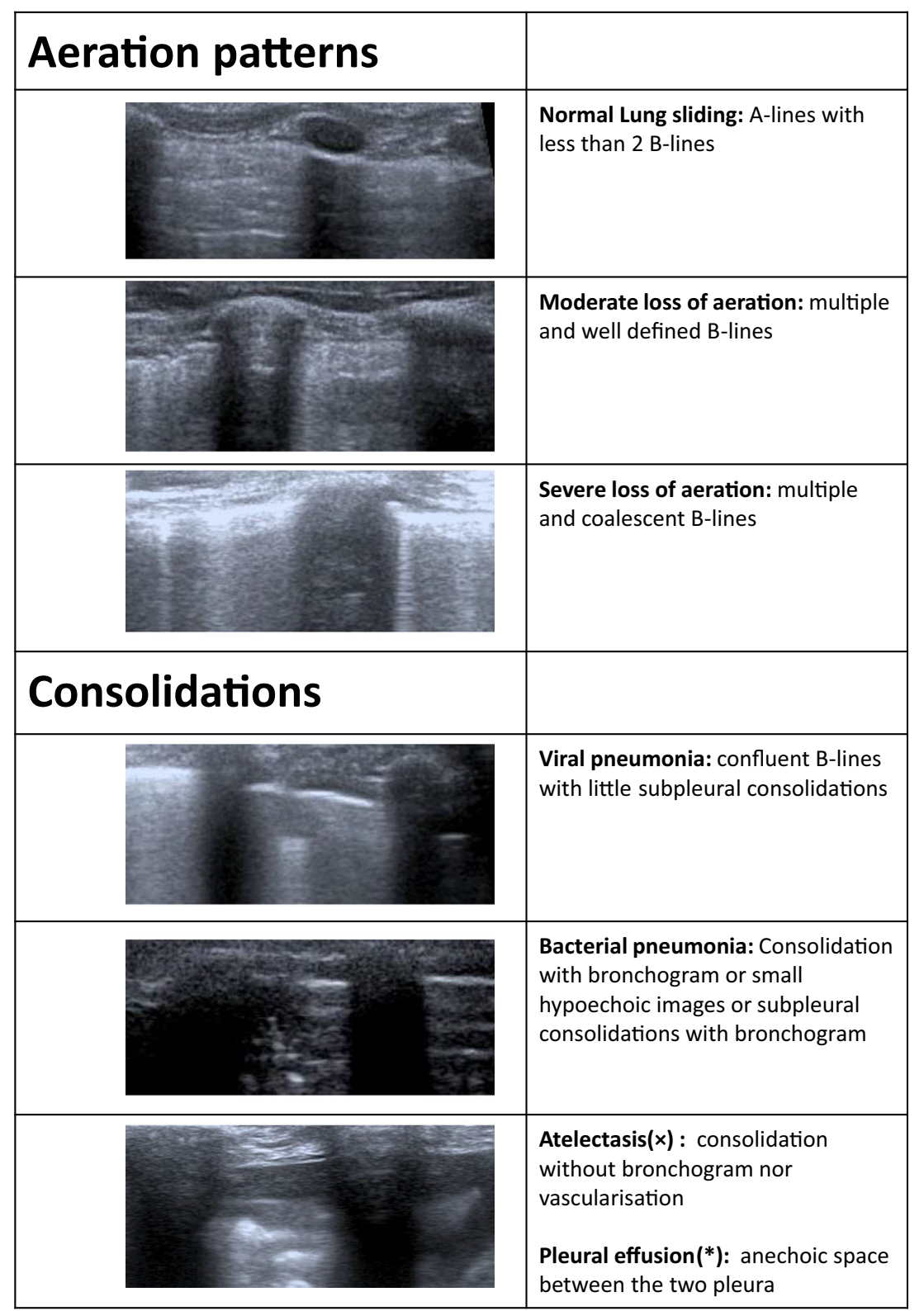


ELISA result with negative PCR for COVID-19. Patients were divided into two groups: there were six patients with respiratory symptoms and ten patients with non-respiratory symptoms. The second group comprised three patients with MIS-C, two with febrile syndromes, one with a urinary tract infection (UTI), one with gastroenteritis, two with lymphadenitis, and one with appendicitis. From the total sample, four patients required PICU admission: two suffering from acute respiratory failure, requiring mechanical ventilation, and two due to MIS-C (Table 1 and Fig. 2). Table 1 summarizes the main characteristics of the patients. A schematic distribution of the patients by their clinical features and the LUS findings is included in Fig. 2. The median time required to carry out LUS was 8 min (IQR 6.5-9). No complications were detected during the procedure.

\section{Patients with respiratory symptoms}

There were $6 / 16$ (37.5\% of the total sample) patients with a positive PCR test for COVID-19 and respiratory symptoms. Four of these patients $(4 / 6,66.6 \%)$ were admitted to the hospitalization ward: three with pneumonia and one with a diagnosis of MIS-C. All four presented mild symptoms, requiring a maximum $\mathrm{FiO}_{2}$ of 0.36 through either a nasal cannula or an oxygen mask. Attending to Soldati et al. score, one patient showed score of 3 , four patients score of 2 , and one patient score of 1 . Regarding the presence and type of consolidation found, 2/4 cases $(50 \%)$ had subpleural consolidation $<1 \mathrm{~cm}$, one had no consolidation (25\%), and the MIS-C patient (25\%) had basal consolidation with a tissue-like pattern without bronchogram and pleural effusion. Two of these patients required PICU admission $(2 / 6,33.3 \%)$ and mechanical ventilation due to respiratory failure. Both of them had moderate-severe aeration loss and subpleural consolidation $<1 \mathrm{~cm}$ on LUS (Fig. 2).

\section{Patients with non-respiratory symptoms}

There were 10/16 (62.5\% of the total sample) COVID-19 patients with other, non-respiratory symptoms; eight of these patients were admitted to the hospitalization ward. Attending to Soldati et al. score, in six patients, LUS showed a score of 2, three patients score of 1 , and one patient score of 0 . LUS showed subpleural consolidation $<1 \mathrm{~cm}$ in two of them (20\%), and consolidation with a tissue-like pattern without bronchogram, in two other patients (20\%). Two of the three (67\%) patients with a MIS-C diagnosis required PICU admission because of their hemodynamic condition. LUS showed a S.score of 2 in both; one had a large consolidation with air bronchograms in the right base of the lung and was eventually diagnosed with a communityacquired bacterial infection (Fig. 2).

\section{Discussion}

During this global respiratory pandemic, LUS has been referred as a key tool in the clinical management of patients with COVID-19-related lung injury [26].

Table 1 Demographic and clinical data

\begin{tabular}{|c|c|c|c|c|}
\hline & Total $(n=16)$ & COVID-19 and RS $(n=6)$ & COVID-19 and n-RS $(n=10)$ & $p$ value \\
\hline Male sex, $n(\%)$ & $11(68.8)$ & $5(83.3)$ & $6(54.5)$ & 0.635 \\
\hline $\begin{array}{l}\text { Age (days) } \\
\text { Median (IQR) }\end{array}$ & $9.4(0.82-12.7)$ & $13.3(10.5-17.2)$ & $2.1(0.33-11)$ & 0.123 \\
\hline Comorbidities & & & & 0.845 \\
\hline None & $13(81.3)$ & $4(66.7)$ & $9(90)$ & \\
\hline Bronchitis & $1(6.3)$ & $1(16.7)$ & $0(0)$ & \\
\hline Oncologic disease & $1(6.3)$ & $1(16.7)$ & $0(0)$ & \\
\hline Congenital malformation & $1(6.3)$ & $0(0)$ & $1(10)$ & \\
\hline $\begin{array}{l}\text { PRISM III } \\
\text { (Median (IQR) }\end{array}$ & $0(0-9.5)$ & $0(0-14)$ & $0(0-2)$ & 0.335 \\
\hline $\begin{array}{l}\text { PICU length (days) } \\
\text { Median (IQR) }\end{array}$ & $7(4-25)$ & $0(0-10)$ & $0(0-1.75)$ & 0.652 \\
\hline $\begin{array}{l}\text { Ward length (days) } \\
\text { Median (IQR) }\end{array}$ & $5(4-9.5)$ & $7(4-15.2)$ & $4(3-7)$ & 0.297 \\
\hline Oxygen therapy $n(\%)$ & $8(50)$ & $6(100)$ & $2(25)$ & $<0.01$ \\
\hline $\mathrm{HFNC} n(\%)$ & $2(12.5)$ & $2(33.3)$ & $0(0)$ & 0.125 \\
\hline NIV $n(\%)$ & $3(18.8)$ & $2(33.3)$ & $1(10)$ & 0.304 \\
\hline CMV $n(\%)$ & $3(18.8)$ & $1(16.7)$ & $2(20)$ & 0.696 \\
\hline Inotropes $n(\%)$ & $3(18.8)$ & $1(16.7)$ & $2(20)$ & 0.696 \\
\hline
\end{tabular}

$R S$, respiratory symptoms; $n$ - $R S$, non-respiratory symptoms; $M I S$, multi-systemic syndrome; $U T I$, urine tract infection; $P I C U$, pediatric intensive care unit; $\mathrm{O}_{2}$, oxygen therapy; $H F N C$, high-flow nasal canula; $N I V$, non-invasive ventilation; $C M V$, conventional mechanical ventilation 


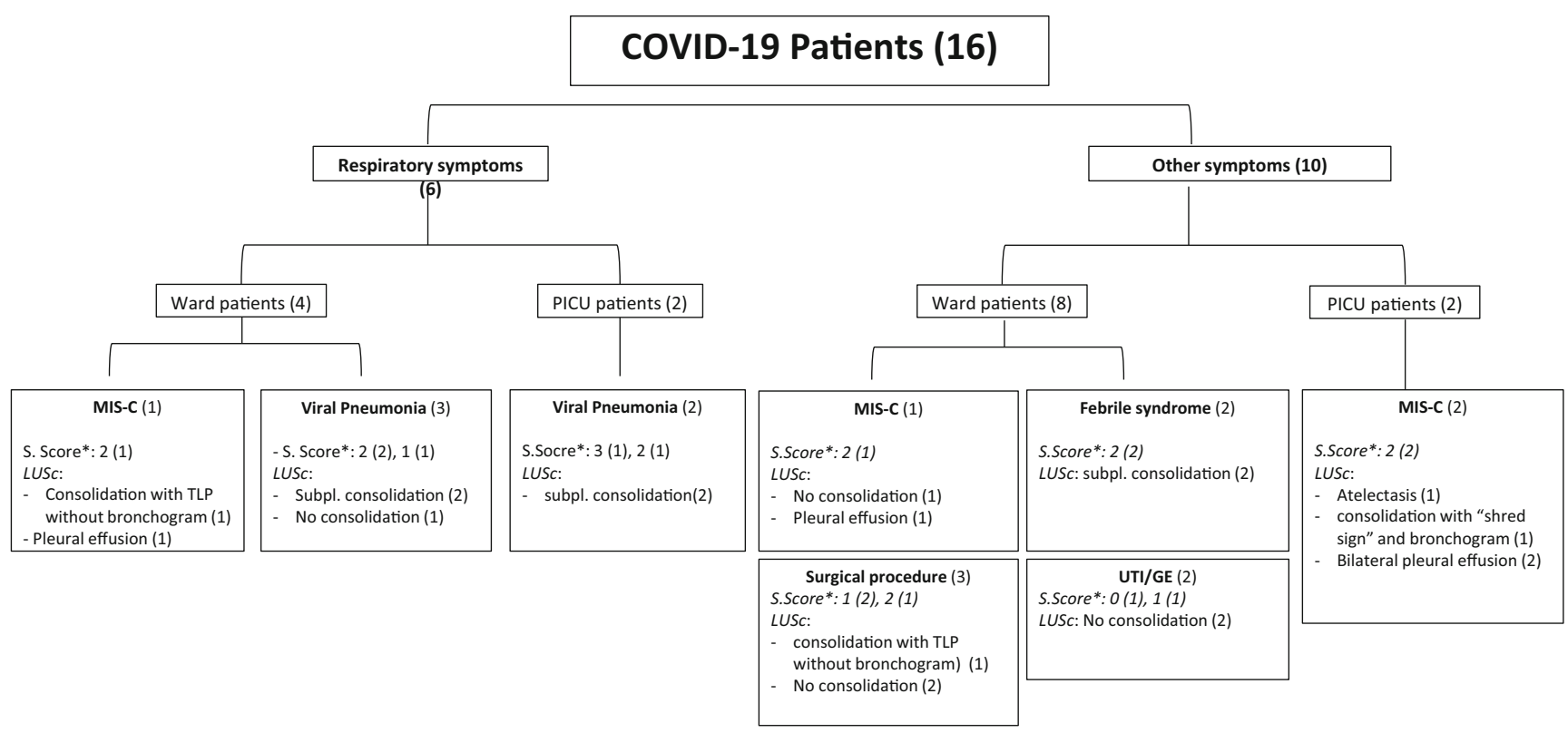

Abbreviations: Sscore: Soldati Score ${ }^{19}$; GE: gastroenteritis; LUSc: lung ultrasound consolidation; MIS: multisystem inflammatory syndrome; PICU: pediatric intensive care unit; Subpl.: subpleural; TLP: tissue-like pattern; UTI: urinary tract infection.

Fig. 2 Distribution of the patients by their clinical features and the LUS findings. Abbreviations: Sscore, Soldati Score [19]; GE, gastroenteritis; LUSc, lung ultrasound consolidation; MIS, multisystem inflammatory

Even though we describe a small sample of pediatric patients, it might be a representative picture of what we could expect to find on the lung ultrasound of children with a COVID-19 infection. In adults, it has been described that LUS findings in COVID-19 patients are similar to those extensively described in patients with other types of pneumonia, including various forms of B-lines, an irregular or fragmented pleural line, consolidations, pleural effusion, and absence of lung sliding [27]. In the COVID-19 patients described in this study, LUS mostly showed multiform vertical artifacts and separate and coalescent B-lines, classified as score of 1 or 2 from Soldati classification. Subpleural consolidations $<1 \mathrm{~cm}$ have been found to be the most predominant pattern, and alveolar consolidation was described in some cases. These results and patterns have been equally defined in adults $[8$, 27]. However, consolidations are less common in our pediatric patients than what is typically described in adults [27].

The classification of both findings seems to be correlated with the severity of the lung injury. A recent paper concludes that there is a high concordance between radiologic and LUS findings, suggesting that LUS is a reasonable method to detect lung abnormalities in children with COVID-19 [20].

Recently, clusters of children and adolescents with a multisystem inflammatory condition sharing features with Kawasaki disease and toxic shock syndrome have been described. In our sample, four patients presented with MIS-C $[16,28-30]$. Even though three of those patients presented with non-respiratory symptoms, LUS showed severe loss of syndrome; PICU, pediatric intensive care unit; Subpl., subpleural; TLP, tissue-like pattern; UTI, urinary tract infection

aeration in all of them. This was probably secondary to the associated capillary leakage, and also to the cardiogenic shock in those who required PICU admission.

LUS findings may differ depending on the presence of respiratory symptoms and their severity. In our experience, we would say that on the one hand, patients with acute COVID-19 disease with severe respiratory failure have a moderately or severely altered LUS, with no cases of a normal or mildly altered LUS. On the other hand, patients with mild or non-respiratory symptoms may have LUS alterations, but milder than those with severe respiratory failure [13, 31]. LUS findings always should be interpreted in light of the clinical context [20].

We would like to mention that we are aware that there may be concern about the safety of using this technique in these patients due to the contagiousness of COVID-19. However, it has been demonstrated that the same clinician can safely perform the clinical examination and the bedside LUS during the same exploration $[13,32]$. Therefore, no other clinicians need to come into contact with the patient [13]. When pulmonary condition can be monitored using LUS, the patient does not need to be moved around the hospital to undergo a CT scan or a chest X-ray, so neither people moving in the corridors nor the radiology technicians come into contact with the patient. All these strategies help to reduce physicians' exposure and halt the spread of the virus. Moreover, combined with the clinical evaluation, LUS may help clinicians to monitor the evolution of lung disease until its resolution [26, 32]. We would like to highlight that no physician got infected despite doing bedside LUS. 
Based on our experience, we consider that LUS might have major utility for the management of children with COVID-19, regardless of whether they have respiratory symptoms, due to its easy access, safety, low cost, and point-of-care use. It could be used to quickly assess the severity of acute COVID-19induced pneumonia, and to track the evolution of the disease during follow-up. It could also allow physicians to identify the patients who are at a higher risk of respiratory failure evolution depending on the LUS alteration, so they could be monitored more intensively and rapidly enhance the treatment if needed.

Recent international evidence-based guidelines on Pointof-Care Ultrasound (POCUS) [33] for critically ill neonates and children have been published. POCUS is increasingly being utilized in neonatal and pediatric critical care as a valuable adjunct to clinical examination. It involves a focused assessment and provides anatomical and/or physiological information to be integrated with clinical and laboratory data and make timely and accurate decisions possible. Lung POCUS is helpful to semi-quantitatively evaluate lung aeration, to detect pneumonia and pleural effusions in neonates and children. Following POCUS guidelines which define a scope of practice may help in standardizing clinical practice across acute care settings. Lung POCUS is helpful to semiquantitatively evaluate lung aeration, to detect pneumonia and pleural effusions in neonates and children.

We acknowledge that this study has several limitations. Firstly, it was carried out at a single center, so the results may not be able to be extrapolated to other populations. Secondly, LUS is a lung-image technique that should be performed by experienced physicians with a standard LUS training and should always be combined with a proper physical examination and other laboratory analysis. Finally, research on COVID-19 pneumonia diagnosis is hampered by the difficulties in obtaining a systematic comparison with $\mathrm{CT}$ scan [10]. Despite this, we believe that it provides valuable information, as there is limited data regarding pediatric patients with this condition.

\section{Conclusions}

LUS could improve how we assess children diagnosed with COVID-19. Two LUS patterns were ascertained: patients with acute respiratory symptoms had a S.score [1] of 2 and 3 with subpleural consolidations $<1 \mathrm{~cm}$. Patients with MIS-C had a S.score of 2. The presence of consolidation on LUS may be due to bacterial cross-contamination. Therefore, LUS can be a useful tool to diagnose and monitor patients throughout their battle with COVID-19.

Acknowledgements This project has been supported by Stavros Niarchos Foundation (SNF), Banco Santander and other private donors of Kidscorona.
Authors' Contributions Dra C. Guitart conceptualized and designed the study, drafted the initial manuscript, and reviewed and revised the manuscript. Drs Suárez, Girona, Bobillo-Pérez, Hernández, and Cambra designed the data collection instruments, collected data, carried out the initial analyses, and reviewed and revised the manuscript. Prof Balaguer and Prof Jordan conceptualized and designed the study, coordinated and supervised data collection, and critically reviewed the manuscript for important intellectual content. All the authors approved the final manuscript as submitted and agree to be accountable for all aspects of the work.

\section{Compliance with ethical statements}

Conflict of interest The authors declare that they have no conflict of interest.

Ethical approval This article does not contain any studies with human participants or animals performed by any of the authors.

Informed consent Informed consent was obtained from all individual participants included in the study.

\section{References}

1. Soldati G, Smargiassi A, Inchingolo R, Buonsenso D, Perrone T, Briganti DF, Perlini S, Torri E, Mariani A, Mossolani EE, Tursi F, Mento F, Demi L (2020) Proposal for international standardization of the use of lung ultrasound for COVID-19 patients; a simple, quantitative, reproducible method. J Ultrasound Med 39:1-7. https://doi.org/10.1002/jum.15285

2. Ghazaly M, Nadel S (2018) Characteristics of children admitted to intensive care with acute bronchiolitis. 913-920. https://doi.org/10. 1007/s00431-018-3138-6

3. Lepri G, Orlandi M, Lazzeri C et al (2020) The emerging role of lung ultrasound in COVID-19 pneumonia. Eur J Rheumatol 7: S129-S133. https://doi.org/10.5152/eurjrheum.2020.2063

4. Pan F, Ye T, Sun P, Gui S, Liang B, Li L, Zheng D, Wang J, Hesketh RL, Yang L, Zheng C (2020) Time course of lung changes on chest CT during recovery from 2019 novel coronavirus (COVID-19) Pneumonia. Radiology 295:200370-200721. https:// doi.org/10.1148/radiol.2020200370

5. Ong JSM, Tosoni A, Kim YJ, Kissoon N, Murthy S (2020) Coronavirus disease 2019 in critically ill children: a narrative review of the literature. Pediatr Crit Care Med 21(C):1-5. https://doi. org/10.1097/PCC.0000000000002376

6. De Rose C, Inchingolo R, Smargiassi A, Zampino G, Valentini P, Buonsenso D (2020) How to perform pediatric lung ultrasound examinations in the time of COVID -19. J Ultrasound Med 39:12. https://doi.org/10.1002/jum.15306

7. Soldati G, Smargiassi A, Inchingolo R, Buonsenso D, Perrone T, Briganti DF, Perlini S, Torri E, Mariani A, Mossolani EE, Tursi F, Mento F, Demi L (2020) Is there a role for lung ultrasound during the COVID-19 pandemic? J Ultrasound Med 39:1-4. https://doi. org/10.1002/jum.15284

8. Peng QY, Wang XT, Zhang LN (2020) Findings of lung ultrasonography of novel corona virus pneumonia during the 2019-2020 epidemic. Intensive Care Med 46(5):849-850. https://doi.org/10. 1007/s00134-020-05996-6

9. Poggiali E, Dacrema A, Bastoni D et al (2020) CLUHCCC in the ED of NC (COVID-19) PRM. 200847. https://doi.org/10.1148/ radiol.202020084. Can lung US help critical care clinicians in the early diagnosis of novel coronavirus (COVID-19) pneumonia? Radiology. 2020 
10. Pata D, Valentini P, De Rose C, De Santis R, Morello R, Buonsenso D (2020) Chest computed tomography and lung ultrasound findings in COVID-19 pneumonia: a pocket review for non-radiologists. Front Med 7. https://doi.org/10.3389/fmed.2020.00375

11. Montes OYA, Danilo B (2020) Routine use of point-of-care lung ultrasound during the COVID-19 pandemic. Med Int. https://doi. org/10.1016/j.medin.2020.04.010

12. Manivel V, Lesnewski A, Shamim S, Genevieve Carbonatto TG CLUE: COVID-19 Lung Ultrasound in Emergency Department. https://doi.org/10.1111/emm.13546

13. Musolino AM, Supino MC (2020) The role of lung ultrasound in diagnosis and follow-up of children with coronavirus disease 2019. Pediatr Crit Care Med (December 2019):1. https://doi.org/10.1097/ PCC.0000000000002436

14. Murray M, Kantilal M, Ruttimann UE (1996) PRISM III. An updated Pediatric Risk of Mortality score. Crit Care Med 24(5):743752

15. Vo P, Kharasch VS (2014) Respiratory Failure. Pediatr Rev 35(11): 476-486. https://doi.org/10.1542/pir.35-11-476

16. America N, America N (2020) Multisystem inflammatory syndrome in children and adolescents temporally related to COVID19. World Health Organisation (WHO). May:13. https://www.who. int/news-room/commentaries/detail/multisystem-inflammatorysyndrome-in-children-and-adolescentswith-covid-19

17. Via G, Storti E, Gulati G, Neri L, Mojoli F, Braschi A (2012) Lung ultrasound in the ICU: from diagnostic instrument to respiratory monitoring tool. Minerva Anestesiol 78(11):1282-1296 http:// www.ncbi.nlm.nih.gov/pubmed/22858877

18. Volpicelli G, Elbarbary M, Blaivas M et al (2012) International evidence-based recommendations for point-of-care lung ultrasound. Intensive Care Med 38(4):577-591. https://doi.org/10. 1007/s00134-012-2513-4

19. Brat R, Yousef N, Klifa R, Reynaud S, Shankar Aguilera S, De Luca D (2015) Lung ultrasonography score to evaluate oxygenation and surfactant need in neonates treated with continuous positive airway pressure. JAMA Pediatr 169(8):1-8. https://doi.org/10. 1001/jamapediatrics.2015.1797

20. Denina M, Scolfaro C, Silvestro E, Pruccoli G, Mignone F, Zoppo M, Ramenghi U, Garazzino S (2020) Lung ultrasound in children with COVID-19. Pediatrics. 146:e20201157. https://doi.org/10. 1542/peds.2020-1157

21. Mongodi S, Santangelo E, De Luca D et al (2020) Quantitative lung ultrasound. Chest. 158(2):469-470. https://doi.org/10.1016/j.chest. 2020.03.080

22. Varshney T, Mok E, Shapiro AJ, Li P, Dubrovsky AS (2016) Pointof-care lung ultrasound in young children with respiratory tract infections and wheeze. Emerg Med J 33(9):603-610. https://doi. org/10.1136/emermed-2015-205302
23. Supino MC, Buonsenso D, Scateni S et al (2019) Point-of-care lung ultrasound in infants with bronchiolitis in the pediatric emergency department: a prospective study. Eur J Pediatr 178(5):623-632. https://doi.org/10.1007/s00431-019-03335-6

24. Lichtenstein D, Mezière G, Seitz J (2009) The dynamic air bronchogram: a lung ultrasound sign of alveolar consolidation ruling out atelectasis. Chest. 135(6):1421-1425. https://doi.org/ $10.1378 /$ chest.08-2281

25. Arbelot C, Ferrari F, Bouhemad B, Rouby J-J (2008) Lung ultrasound in acute respiratory distress syndrome and acute lung injury. Curr Opin Crit Care 14(1):70-74. https://doi.org/10.1097/MCC. 0b013e3282f43d05

26. Smith MJ, Hayward SA, Innes SM, Miller A (2020) Point-of-care lung ultrasound in patients with COVID-19 - a narrative review. Anaesthesia. 75:1096-1104. https://doi.org/10.1111/anae.15082

27. Volpicelli G, Lamorte A, Villén T (2020) What's new in lung ultrasound during the COVID-19 pandemic. Intensive Care Med 46:1445-1448. https://doi.org/10.1007/s00134-020-06048-9

28. Riphagen S, Gomez X, Gonzalez-Martinez C, Wilkinson NTP (2020) Hyperinflammatory shock in children during COVID-19 pandemic. Lancet. 395:1607-1608

29. DeBiasi RL, Song X, Delaney M, Bell M, Smith K, Pershad J et al (2020) Severe COVID-19 in children and young adults in the Washington, DC Metropolitan Region. J Pediatr 223:199-203.e1. https://doi.org/10.1016/j.jpeds.2020.05.007

30. Jones VG, Mills M, Suarez D, Hogan CA, Yeh D, Bradley Segal J et al (2020) COVID-19 and Kawasaki disease: novel virus and novel case. Hosp Pediatr 10:537-540

31. Musolino AM, Supino MC, Buonsenso D, Ferro V, Valentini P, Magistrelli A, Lombardi MH, Romani L, D'Argenio P, Campana A, Roman Lung Ultrasound Study Team for Pediatric COVID-19 (ROMULUS COVID Team) (2020) Lung ultrasound in children with COVID-19: preliminary findings. Ultrasound Med Biol 146(1):2094-2098. https://doi.org/10.1016/j.ultrasmedbio.2020. 04.026

32. Buonsenso D, Pata D, Chiaretti A (2020) COVID-19 outbreak: less stethoscope, more ultrasound. Lancet Respir Med 8(5):e27. https:// doi.org/10.1016/S2213-2600(20)30120-X

33. Singh $Y$, Tissot $C$, Fraga MV et al (2020) International evidencebased guidelines on Point of Care Ultrasound (POCUS) for critically ill neonates and children issued by the POCUS Working Group of the European Society of Paediatric and Neonatal Intensive Care (ESPNIC). Crit Care 24(1):65. https://doi.org/10.1186/s13054-0202787-9

Publisher's note Springer Nature remains neutral with regard to jurisdictional claims in published maps and institutional affiliations. 